\title{
COMMENT
}

Check for updates

\section{Overcoming vaccine hesitancy in low-income and middle-income regions}

Clarissa Simas $\mathbb{1}^{1 凶}$ and Heidi J. Larson ${ }^{1,2}$

Vaccine hesitancy is prevalent in all demographic groups and settings; however, it remains vastly understudied in low-income and middle-income regions. Successful strategies to address hesitancy require an understanding of regional, cultural, and economic factors, and tailored training of health-care professionals. Vaccine confidence crises should be addressed swiftly. Further research is needed to optimize approaches.

Vaccinations are one of the most successful and costeffective public health measures and are estimated to prevent 4-5 million deaths per year; yet, vaccines continue to face scepticism. Concerns over their safety and efficacy, often due to unfounded rumours, lead individuals and entire groups to delay or refuse vaccines. The WHO recently named vaccine hesitancy as one of the top threats to global health ${ }^{1}$.

Vaccine hesitancy is prevalent in rich and poor regions alike and can be found among individuals of various socioeconomic, cultural, ethnic and religious backgrounds. Hesitancy can stem from marginalization and social exclusion, negative experiences at health services, misinformation about vaccines circulated on unregulated social media platforms, or a lack of trust in authorities and institutions. Determinants of vaccine hesitancy can be grouped into vaccine-specific issues, individual and group influences, or other contextual factors (for example, historical or political influences) ${ }^{2}$.

In low-income and middle-income countries (LMICs), vaccination is generally accepted, with pockets of reduced acceptance in some communities ${ }^{3}$. In $2018,>90 \%$ of a representative population sample in South Asia and East Africa perceived vaccination as effective and safe ${ }^{4}$. However, despite decades of vaccine programme success through multilateral initiatives, such as Gavi, the Vaccine Alliance, amongst others, scepticism and concern about vaccines have increased in LMICs in recent years, which has left entire populations vulnerable to disease. The COVID-19 pandemic has worsened this situation through the increased spread of misinformation and disinformation about the safety and efficacy of vaccines. In 2020, a 15-country study conducted by the Africa Centres for Disease Control and Prevention showed high variation in the share of population who said they would be willing to vaccinate against COVID-19, ranging from $94 \%$ in Ethiopia to only $59 \%$ in the Democratic Republic of Congo ${ }^{5}$. The underlying causes of vaccine hesitancy in LMICs vary considerably between regions and vaccines.
Vaccination drives in Africa have made remarkable advances in the past decade, notably an increased coverage of childhood vaccines and a substantial decline in measles mortality and poliomyelitis incidence. Yet, controversies have led to delays or refusal of vaccination in the region. One notable example is the boycott to poliomyelitis immunization in Nigeria, driven by rumours and distrust, which lead to poliomyelitis outbreaks across three continents ${ }^{6}$.

In Latin America and the Caribbean, negative beliefs and attitudes towards immunization are the most commonly reported barrier to vaccination uptake, irrespective of the vaccine $e^{7}$. The main reason for missed vaccinations among children in this region is a lack of information provided by health-care professionals and health authorities, which leads to rumours and false beliefs around the safety and efficacy of childhood vaccines ${ }^{7}$. The dissemination of information about how vaccination benefits far outweigh its risks can help to mitigate these concerns, whereas misinformation amidst eroding trust can create a fertile ground for vaccine confidence crises.

The suspension of the Dengvaxia vaccine against Dengue disease in the Philippines is an example of how a highly publicized vaccine confidence crisis can have damaging effects on population health. Dengvaxia was one of the first vaccines developed especially for use in LMICs and one that has the potential to greatly decrease disease mortality. One year after introduction, the manufacturer published a warning of a slightly increased risk of acute Dengue disease in individuals who have not had Dengue infection before vaccination. This warning led to accusations by various parties that vaccines caused deaths and that Filipino health authorities were corrupt ${ }^{8}$. This situation highlights the importance of appropriate risk communication strategies.

Approaches to address vaccine hesitancy can be dialogue-based (involving religious or traditional leaders, social media, mass media or direct communication between health-care professionals and individuals), 


\section{Box 1 Strategies to address vaccine hesitancy}

- Successful strategies require an understanding of regional, cultural, and economic factors.

- Health-care professionals should be trained to address vaccine hesitancy among patients.

- Emerging vaccine confidence crises should be addressed early.

- Communication and outreach strategies should be tailored to the relevant groups and the specific vaccines.

- Politicians and health authorities should refrain from politicizing vaccine debates.

incentive-based (for example, provision of goods to encourage vaccination) and reminder-based or recall-based (letters and phone calls sent to target populations). Vaccine hesitancy has many layers, and no single approach can fully address it (BOX 1). Interventions that involve various strategies and focus on dialogue-based approaches tend to perform best ${ }^{9}$. Health authorities should listen to concerns and engage with rumours upfront to prevent an erosion of vaccine trust, particularly in regions with long histories of systematic neglect by health systems and officials. This recommendation also applies to high-income regions, which are not immune to confidence crises if concerns and scepticism go unchecked.

Confidence and attitudes towards different vaccines in LMICs remain understudied ${ }^{7}$, and more research is needed to tailor approaches to different regions and also to different vaccination programmes (that is, childhood, maternal, or adult). For example, although vaccine recommendations by a health-care professional are a strong indicator of maternal vaccine uptake globally, the influence of spouses and partners in the decision-making process can vary greatly ${ }^{10}$. A tailored strategy could aim at building confidence in maternal vaccines among spouses. Research and in-depth analyses are necessary to understand local sentiments, beliefs and behaviours associated with immunization.

To improve vaccine confidence and uptake, training health-care professionals to address hesitancy is crucial. Health-care professionals are often confronted with vaccine hesitancy and have to manage difficult conversations, appease anxieties and respond to concerns. Training must elicit their confidence and prepare them to listen empathetically, properly inform patients and be open to questions, including those about vaccine safety.

Communication strategies, including how to explain risks, should consider local histories, engage hesitant groups, and work together with trusted brokers from communities to build confidence in vaccines. Messages via any medium that communicate facts while being engaging, empathetic and touching on positive emotions are important. Health authorities should be prepared to quickly and directly address any confidence crises by listening to concerns, before the crises become uncontrollable. These recommendations remain part of wider efforts to build vaccine confidence. Moreover, in a world hyper-connected by social media, vaccine concerns and anxieties are no longer bounded by geography. One example is the diffusion of vaccine scepticism from France to francophone Africa. Echoes of vaccine hesitancy coming from social media should not be ignored, and online conversations must be paid attention to.

Lastly, health authorities and political figures have the most important roles and responsibilities in addressing vaccine hesitancy. It cannot be emphasized enough that authorities and politicians should refrain from polarizing or politicizing vaccine debates and should endorse vaccination instead, while setting up and ensuring funding for initiatives that guarantee access for all.

1. World Health Organization. Ten threats to global health in 2019 who int https://www who int/news-room/spotlight/ten-threats-toglobal-health-in-2019 (2019).

2. MacDonald, N. E. et al. Vaccine hesitancy: Definition, scope and determinants. Vaccine 33, 4161-4 (2015).

3. Bhopal, S. \& Nielsen, M. Vaccine hesitancy in low- and middle-income countries: Potential implications for the COVID-19 response. Arch. Dis. Child. 106, 113-114 (2021).

4. Wellcome Trust. Wellcome Global Monitor 2018 Report. Perceived safety and effectiveness of vaccines by regions. wellcome.ac.uk https://wellcome.ac.uk/reports/wellcome-global-monitor/2018 [Accessed May 15 2021].

5. Africa Centres for Disease Control and Prevention. COVID 19 Vaccine Perceptions: A 15 country study. February 2021. africacdc. org https://africacdc.org/download/covid-19-vaccine-perceptions-a15-country-study/ (2021)

6. Ghinai, I., Willott, C., Dadari, I. \& Larson, H. J. Listening to the rumours: What the northern Nigeria polio vaccine boycott can tell us ten years on. Glob. Public Health 8, 1138-1150 (2013).

7. Guzman-Holst, A., DeAntonio, R., Prado-Cohrs, D. \& Juliao, P. Barriers to vaccination in Latin America: a systematic literature review. Vaccine 38, 470-481 (2020).

8. Dayrit, M. M., Mendoza, R. U. \& Valenzuela, S. A. The importance of effective risk communication and transparency: lessons from the dengue vaccine controversy in the Philippines. J. Public Health Policy 41, 252-267 (2020).

9. Jarrett, C., Wilson, R., O'Leary, M., Eckersberger, E. \& Larson, H. J. Strategies for addressing vaccine hesitancy - a systematic review. Vaccine 33, 4180-4190 (2015).

10. Wilson, R. J., Paterson, P., Jarrett, C. \& Larson, H. J. Understanding factors influencing vaccination acceptance during pregnancy globally: a literature review. Vaccine 33, 6420-6429 (2015).

\section{Competing interests}

C.S. is involved in collaborative grants with GlaxoSmithKline and Johnson $\&$ Johnson. H.J.L. is involved in collaborative grants with GlaxoSmithKline, Johnson $\&$ Johnson and Merck and has also received other support for participating in Merck meetings and GlaxoSmithKline advisory roundtables; H.J.L. is a member of the Merck Vaccine Confidence Advisory Board. 